\title{
LA IMPORTANCIA DEL DINERO EN LA ELECCIÓN LABORAL
}

The importance of money in labor choice

\section{José Vicente Pascual Ivars* Roberto Luna Arocas ${ }^{*}$}

Resumen: El objetivo fundamental del presente artículo es identificar los factores motivadores del trabajo entre los futuros directivos y trabajadores y reflexionar desde un punto de vista tanto académico como profesional sobre la importancia del dinero como factor motivador. Para ello, hemos tomado a estudiantes universitarios, en su faceta de futuros trabajadores; se concluye que el dinero por sí solo no motiva y que es necesario encontrar aquel grupo de factores que motivan a los trabajadores con el objetivo de conseguir el máximo rendimiento. Los resultados nos permiten concluir que no debemos motivar tan solo con el dinero o admitiéndolo como principal elemento motivacional.

Palabras clave: Dinero, motivación, factor motivacional, satisfacción laboral, compromiso.

José Vicente Pascual Ivars. Es licenciado en Ciencias Económicas y Empresariales y doctor en Dirección de Empresas, Estrategia y Organización por la Universidad de Valencia, España. Email: jose.pascual@uv.es

** Roberto Luna Arocas. Profesor del Departamento de Dirección de Empresas de la Universidad de Valencia. Email: Roberto.luna@uv.es 
Abstract: The main aim of this paper is to identify the motivation factors among future managers and workers, and to analyze from both an academic and professional point of view, about the importance of money as a motivating factor. To do this, we interviewed college students, in their role as future workers, concluding that money by itself does not motivate workers and that we need to find the bundle of factors that motivate employees in order to achieve a better performance. The results allow us to conclude that we should not only motivate with money or with money as the primary motivational element.

Keywords: Money, motivation, motivational factors, job satisfaction, commitment.

\section{Introducción}

En una sociedad donde el sector terciario cobra cada vez más importancia y donde se ha demostrado que el factor humano es probablemente el más importante en la consecución del éxito por parte de las empresas, parece lógico, que se analice cuáles son los factores que motivan a un trabajador para que acepte o rechace un determinado trabajo. El análisis desde un punto de vista científico de las razones por las que un trabajador decide trabajar o no puede arrojar luz sobre las motivaciones que tiene en el desempeño diario de su trabajo y, por ende, de los factores que harán que fracase o tenga éxito en su trabajo.

Al realizar una revisión de la literatura científica que analiza los factores que motivan a un trabajador a aceptar un trabajo y no otro, encontramos también numerosos trabajos que analizan también la satisfacción laboral y el compromiso organizacional. 
Probablemente la referencia más importante y más citada que podemos encontrar proviene del trabajo realizado por Herzberg et al. (1959), el cual tiene como punto de partida una muestra heterogénea desde el punto de vista sociodemográfico, los referidos autores analizaron una serie de factores motivacionales como la seguridad, el interés, la posibilidad de promocionar, el sentirse valorado, entre otros. Los autores solicitaron a los encuestados que ordenasen estos factores por orden de importancia para, finalmente, fruto de esos numerosos estudios realizados elaborar una lista de los catorce factores más importantes.

La teoría de Herzberg, denominada también como teoría de los dos factores ha sido utilizada en numerosas ocasiones por la literatura científica además de Harpaz (1990) y Luna-Arocas y Tang (2004) destacan autores como Cheng (2007), quienes la utilizan en un sentido positivo para justificar que a pesar de las diferencias culturales los factores higiénicos y motivadores afectan de forma similar a lo largo del planeta. En su artículo Bassett-Jones y Lloyd (2005) se preguntan si 50 años después, la teoría de Herzberg puede tener vigencia. Los autores concluyen, a pesar del trascurso del tiempo y tras encuestar a 3,200 personas que asocian con esta teoría los factores vinculados con motivaciones intrínsecas que juegan un papel destacadamente más importante. A continuación realizaremos el análisis de una serie de líneas de investigación que al igual que las citadas en este párrafo parten del estudio de Herzberg para medir, de una forma u otra, la satisfacción laboral.

Esta línea de investigación continuó con los estudios de Weiss, Dawis, England y Lofquist (1964) quienes elaboraron un cuestionario a partir de factores de los que dependía la satisfacción laboral. En el año 1971, Lawler analiza (al igual que Luna-Arocas y Tang (2004), en la posterioridad, analizó la importancia del salario llegando a la conclusión de que aparecía en tercer lugar según el orden de importancia. Sin embargo y en contra de lo demostrado hasta el momento Quinn (1971), utilizando una muestra de trabajadores analizó entre otros factores 12 de los 14, llegó a la 
conclusión de que ninguno de los factores destacaba en importancia por encima de los demás de manera significativa.

Vroom (1964), introdujo como novedad el establecimiento de una conexión (teoría de las expectativas) entre la importancia que los trabajadores otorgan a cada uno de los diferentes aspectos laborales y como afectan a nivel global cada uno de esos factores a la satisfacción laboral. En este sentido, Lawler (1973) en esta misma línea concluye que para la mayoría de trabajadores la satisfacción salarial tiene una gran influencia sobre la satisfacción global.

Rathankoon y Ogulama (2003) intentan aplicar la teoría de Herzberg al mercado Tailandés de la construcción. Para ello utilizan catorce factores utilizados por Herzberg, como son: el logro, el reconocimiento, el trabajo en sí mismo, la responsabilidad, el avance o la mejora, la posibilidad de crecimiento, la administración y políticas empresariales, la supervisión técnica, las relaciones con los supervisores, las condiciones laborales, el salario, las relaciones con los iguales, la vida personal, las relaciones con los subordinados, es estatus, la seguridad laboral, los accidentes y las relaciones con otras empresas. Los resultados indican que en este caso, el logro es el factor más mencionado como fuente de satisfacción en las entrevistas informales realizadas. La importancia del factor reconocimiento destaca por ser inferior que en el estudio de Herzberg. La responsabilidad y el adelanto puntúan parecido que en el estudio de Herzberg. La administración y las políticas empresariales aparecen equilibradas mientras que para Herzberg esta era la principal fuente de insatisfacción. El salario es el factor mejor puntuado de aquellos relacionados con el puesto de trabajo, lo que significa que para los trabajadores tailandeses de la construcción tiene cierta importancia. Aunque el salario también adquiere cierta relevancia como factor insatisfactor.

Las relaciones sociales adquieren gran importancia en los dos grupos de factores, es decir, pertenecerían a ambos grupos de 
factores para estos trabajadores, mientras que para Herzberg son factores higiénicos. Las condiciones laborales coinciden con lo expuesto por el teórico de los dos factores apareciendo como un factor higiénico, aunque en el presente estudio su influencia es menor. La supervisión contradice la teoría analizada ya que parece inclinarse como factor motivador en lugar de factor higiénico. Con lo que podemos concluir que la clasificación establecida por Herzberg de factores satisfactores e insatisfactores no se cumple al cien por cien ya que existen factores que contradicen al autor en ambos sentidos según el estudio realizado con trabajadores de la construcción en Tailandia.

Podemos comprobar cómo esta línea de investigación sigue abierta y sigue aportando nuevas conclusiones como puede verse en el trabajo de Rynes, Gerhart y Minette (2004) quienes analizan la importancia del dinero como factor motivador y la diferencia existente entre el valor que los trabajadores le otorgan y el que declaran que tiene para ellos. En este estudio, los autores concluyen que el dinero no es el único factor motivador y que por supuesto tampoco no es el primero para todo el mundo. Pero confirman la evidencia de que es un factor importante para la mayoría de la gente. Ahondando más en el tema, los autores concluyen que hay una evidencia que queda manifiesta en los estudios donde se pide a los trabajadores que ordenen el dinero y otros motivadores: el hecho de que los cambios a nivel salarial tienen una importancia y que la forma como se determina actualmente el salario afecta a las decisiones de seguir o no en las empresas. Finalmente, concluyen que deben combinarse tanto factores monetarios, como no monetarios para lograr que el trabajador se sienta motivado.

Hay estudios que analizan la satisfacción de los directivos, como el realizado por Porter (1964) a través del cual llegó a la conclusión de que para los directivos las necesidades de rango superior (las necesidades sociales, de estima, la autonomía y la autorrealización) son las más importantes aunque se encontraban más satisfechos con las de orden inferior (seguridad, salario). En la misma línea 
encontramos el estudio de Porter y Lawler (1965) donde se concluye que para los trabajadores esas necesidades primarias adquieren más importancia que para los directivos. En este mismo sentido, Garrido, Pérez y Antón (2005) analizan la satisfacción laboral de los directores o jefes de ventas y llegan a la conclusión de que las prácticas de recursos humanos basadas en la compensación (niveles y tipos) y en el diseño del puesto de trabajo (recursos y autonomía) resultan ser "las principales culpables" de la satisfacción laboral de los jefes de ventas. Al mismo tiempo, los autores confirman que existe una creciente necesidad para las empresas de combinar incentivos económicos con factores no económicos para lograr la satisfacción de los directivos.

Hay estudios que han utilizado muestras tanto de directivos como de trabajadores, como el caso de Dunn (2001), quien realiza un estudio en organizaciones con estructura matricial. Utiliza para ello factores tomados de Herzberg como son: factores higiénicos (el salario, las relaciones con el superior, la supervisión técnica, la administración y políticas organizativas, las condiciones laborales, los factores de la vida personal, el estatus y la seguridad) y factores motivadores (el reconocimiento, el logro, la responsabilidad, el trabajo en sí mismo, el aprendizaje y las posibilidades de crecimiento) dejándose fuera del estudio las relaciones interpersonales tanto con subordinados como con compañeros.

El estudio fue realizado partiendo de tres muestras diferentes: los directivos funcionales, los jefes o directores de equipo y miembros de equipos de proyecto. Los resultados arrojados corroboran la existencia de un patrón de control para los miembros de equipos de proyecto en una organización matricial. Otra conclusión a la que llegó el autor fue que mientras que los jefes de proyecto intentan controlar los factores motivadores, los jefes funcionales intentan controlar las variables higiénicas. Con este mismo tipo de muestra combinada, Leach y Westbrook (2000) fundamentaron su estudio, sobre un laboratorio de investigación perteneciente al gobierno norteamericano, con la teoría de factores de Herzberg. Para ello 
tomaron datos tanto de investigadores como de directivos del laboratorio. Los autores utilizaron en el mismo orden diez factores utilizados por Herzberg que a continuación detallamos: en primer lugar (y por orden los factores motivadores): (logro, reconocimiento, el trabajo en sí mismo, responsabilidad y promoción) y, en segundo lugar, los factores higiénicos (la administración y políticas de la empresa, la supervisión técnica, el salario, las relaciones interpersonales y las condiciones laborales). Los autores plantearon dos preguntas a los encuestados, la primera les preguntaba cuáles de los diez factores les hacían sentir motivados; cada uno de los diez factores incluía una breve explicación en línea con el sentido transmitido por Herzberg, excepto la explicación sobre la promoción que fue sustituida por la explicación del trabajo de Herzberg (1966), donde aclara que se supone que con un aumento de tareas es suficiente, aclarando así que no es necesario un cambio en el estatus. La segunda pregunta les solicitaba a los encuestados que pensaran en momentos donde se sentían especialmente mal o insatisfechos con su trabajo. Se les solicitaba que puntuasen de nuevo la importancia de cada uno de esos diez factores.

Finalmente, se les formuló una tercera pregunta con relación a si en ese momento se sentían felices y satisfechos o si por el contrario se sentían insatisfechos en su trabajo. En las tres preguntas los autores dejaban un cierto espacio para que los encuestados pudiesen redactar también sus opiniones. Los resultados arrojaban las siguientes consideraciones: respecto a la primera pregunta (factores motivadores), los factores más importantes son, en los dos casos, los motivadores excepto las relaciones interpersonales situadas por encima de lo previsto por los autores. Otro resultado es que tanto los directivos como los investigadores coinciden en los tres factores considerados como más importantes (logro, trabajo en sí mismo y el reconocimiento) y que coincidirían en el orden de importancia excepto en dos factores (los investigadores consideran, en cuarto lugar, las relaciones interpersonales y, en quinto lugar, la responsabilidad, mientras que en el caso de los directivos 
es lo contrario; el segundo cambio reside en que mientras que los directivos valoran la supervisión técnica en octavo lugar y las condiciones laborales en noveno, los investigadores lo hacen al revés).

Respecto a la segunda pregunta (factores que provocan insatisfacción), los resultados obtenidos por los autores confirman lo expuesto por Herzberg y son los factores higiénicos los que ocupan los primeros lugares en importancia. En esta segunda cuestión los directivos y los investigadores no parecen coincidir tanto, aunque destaca el hecho de que tanto para un grupo como para el otro las condiciones laborales y la administración y políticas empresariales puntúan en los primeros lugares. También destaca el hecho de que los directivos consideren el salario como el último factor que provoca insatisfacción, mientras que los investigadores lo consideran el cuarto factor más importante. Finalmente, en lo referente a la tercera pregunta formulada (situación actual), se les proponían tres categorías: insatisfechos, neutrales y motivados. El 37\% de los encuestados se sentían insatisfechos o neutrales. El número de investigadores insatisfechos supera al de directivos.

Respecto a la comparación con los resultados obtenidos por Herzberg prácticamente coinciden y según los autores coincidirían aún más de no ser por la administración y políticas empresariales y las relaciones interpersonales que diferían significativamente del orden propuesto por Herzberg.

La teoría de Herzberg ha sido utilizada como base de análisis de la satisfacción de estudiantes en las universidades, para medir la satisfacción del aprendizaje mediante herramientas virtuales y en la medición de la satisfacción laboral (Tietjen, 1998; Tang, 1993; Luna y Tang, 2004), entre otros.

Cheng (2007) analiza la satisfacción de estudiantes universitarios (en situación laboral) con la finalidad de analizar su motivación por estudiar y dotar a los administradores del programa (es un 
programa público) de los conocimientos sobre los motivadores de los estudiantes. Para ello se basó en la tipología de Herzberg de factores higiénicos (política organizacional, salarios, relaciones con los compañeros, beneficios del trabajo, condiciones laborales, relaciones con jefes y subordinados, estabilidad laboral, fondos de garantía por jubilación...), y de factores motivadores (el crecimiento personal, la pasión por el trabajo, la responsabilidad social, la oportunidad de avanzar o promocionar y el sentimiento de realización o logro). El autor intenta, basándose en estos factores, adaptarlos a su contexto, utilizando así dieciséis factores, como el crecimiento personal, el gusto por aprender, porque los amigos me lo pidieron, el profesor tiene prestigio (relaciones con los demás), por aprender...

El estudio concluye que los principales motivadores de los estudiantes (de tercer ciclo y máster y de los últimos cursos universitarios) son: la creación de ventajas propias (destacar positivamente), la necesidad de sentirse reconocido, el gusto por aprender, el plan de estudios o programa de cursos, la reputación de la institución, el crecimiento personal y la demanda de las nuevas economías. Por otro lado, esta investigación también muestra que los factores higiénicos como la política organizacional, los nuevos amigos, las relaciones con subordinados, la presión por esforzarse y la autoridad como directivo en el lugar de trabajo también resultaron significativas. El autor no encontró diferencias significativas en lo referente al género o a la edad.

Con relación a los diferentes estudios que analizan la satisfacción desde un punto de vista grupal existen dos líneas fundamentales, básicamente contrapuestas: por un lado, aquellos que afirman que existen diferencias significativas entre los grupos a la hora de valorar la importancia de las diferentes facetas laborales (Friedlander, 1965; Hinrichs, 1968; Hofstede, 1972) y, por otro lado, los autores que han encontrado similitudes significativas entre grupos en la valoración de la importancia de los diferentes aspectos (Ronan, 1970; Stracevich, 1972). 
La siguiente corriente investigadora que cabe destacar relacionada con los aspectos motivacionales del trabajo es aquella que estudia las diferencias y similitudes a la hora de valorar los diferentes factores entre países. En este sentido, hay autores que han encontrado similitudes significativas en la valoración de la importancia de los factores motivacionales entre los diferentes países (Haire et. al., 1966; Sirota y Greenwood, 1971). En este mismo sentido Kraut y Ronen (1975) encontraron pocas diferencias en la valoración de la importancia de las diferentes facetas motivacionales entre los diversos países. Así Bigoness y Hofstede (1987) que recabaron información sobre motivaciones laborales de trece grupos (en dos momentos diferentes del tiempo) concluyeron y demostraron que la importancia de diez facetas motivacionales y la permanencia estable en el transcurso del intervalo de tiempo. En ambos estudios los encuestados puntuaban en orden de importancia el trabajo con retos, la responsabilidad o libertad, la buena relación con el inmediato superior y las posibilidades de promocionar como los cuatro factores más significativos.

En contraposición a estos autores hay una corriente de autores que han encontrado divergencias y diferencias entre las diferentes naciones a la hora de valorar los diferentes objetivos motivacionales. De esta forma, Bass y Eldridge (1979) hallaron resultados que concluían que directivos con éxito en Dinamarca hacían hincapié en la importancia de los aspectos sociales a la hora de tomar decisiones, en contraposición a los directos americanos, británicos y alemanes que valoraban como aspecto a destacar los beneficios económicos. En esta misma línea, Hofstede (1980) en un estudio realizado para una empresa con sede en cuarenta países concluyó que había diferencias significativas en cuatro dimensiones culturales, a las que denominó distancia en el poder, aversión a la incertidumbre, individualismo y masculinidad. Según Kunnungo y Wright (1983), los directivos británicos otorgaban una mayor importancia a la autonomía y al logro personal que los directivos franceses. Los franceses valoraban una supervisión competente, 
políticas empresariales sólidas, retribución extra o reparto de beneficios, seguridad y unas buenas condiciones laborales. Dowling y Nagel (1986) concluyeron que los encargados australianos hacían cierto énfasis en los aspectos extrínsecos, mientras que sus homólogos americanos valoraban el enriquecimiento personal, la responsabilidad y otros factores intrínsecos, como los más destacados.

En este mismo sentido encontramos el estudio de Beer y Katz (2003) que justifican diferencias entre países aunque para ello los autores se centran en el análisis del papel de los incentivos (concretamente de los bonus). Las principales diferencias que los autores encuentran residen en que la consideración de los incentivos en el caso de los directivos es un constructo social que prevalece más en los Estados Unidos que en Europa o Asía. Los autores encontraron que por norma general los ejecutivos opinan que los bonus motivan aunque mejoran muy poco el rendimiento si es que lo llegan a mejorar. Otro resultado que hallaron los autores es el hecho de que el uso de bonus se justifica por su papel como atrayentes y retenedores de ejecutivos (es decir, volviendo al concepto explicado al principio, actúan como motivadores). Los autores concluyen que los incentivos como parte de la retribución pueden ser utilizados para retener y atraer a los ejecutivos, con lo que pueden utilizarse para motivar, aunque con muchas limitaciones.

Finalmente, analizamos las investigaciones que estudian la influencia del género en la valoración de los diferentes factores motivacionales. Los resultados analizados parecen confirmar la tan frecuentemente citada generalización de que las mujeres están más orientadas que los hombres hacia los aspectos interpersonales de sus trabajos (Hardin et al., 1957; Kilpatrickmas et al., 1964; Centres y Bugental, 1966). 


\section{La importancia del dinero y la actitud de los estudiantes hacia el dinero}

El principal objetivo de este artículo es analizar hasta qué punto la importancia del dinero modera y condiciona al resto de factores motivadores. Estudiaremos la actitud hacia el dinero de los estudiantes, en su papel como futuros trabajadores, y su relación con los factores motivadores. Este estudio analiza la importancia del dinero como factor moderador de la relación entre la satisfacción laboral y el compromiso organizacional. La importancia que las personas, como trabajadores, otorgamos al dinero va a tener una influencia significativa tanto en el compromiso organizacional como en la satisfacción laboral (Luna-Arocas \& Artacho Ruiz, en prensa). Estudiamos el dinero como factor extrínseco en el proceso de satisfacción. El dinero es considerado como un factor higiénico (extrínseco) y no como un factor motivador (intrínseco). (Hertzberg, Mausner \& Snyderman, 1959).

En este artículo pretendemos demostrar que el dinero es importante como factor motivador, pero aún siendo así no es ni el único, ni siquiera el más importante tal y como podemos comprobar en la literatura (Harpaz, 1990, Pascual-Ivars, 2006, Luna y Tang, $2004 . .$.$) , circunstancia que se demostró en siete países diferentes,$ en diferentes niveles organizativos, con independencia del género y de la edad (Harpaz, 1990).

En este estudio pretendemos comprobar si se confirman los hallazgos encontrados hasta el momento en la literatura del tema en los estudiantes universitarios o, si por el contrario, los estudiantes son un caso diferente.

\section{La influencia del dinero en el sistema retributivo}

En esta investigación pretendemos analizar la actitud hacia el dinero de los estudiantes a través de la propuesta de tres escenarios 
retributivos diferentes. En estos tres escenarios les proponemos diferentes combinaciones entre retribución fija y variable. La elección del escenario vendrá condicionada no solo por la importancia que le otorguen al dinero sino también por su mayor propensión/aversión al riesgo. Aquellas personas más adversas al riesgo escogerán un escenario más conservador compuesto principalmente o únicamente por una retribución de carácter fijo. Eso por un lado, por otro aquellas personas más propensas al riesgo escogerán un escenario con una mayor proporción de retribución variable que los adversos al riesgo. Al mismo tiempo analizaremos cómo el dinero condiciona la elección del escenario independiente de la aversión o propensión al riesgo.

\section{Las diferencias entre los factores motivadores esperados y los deseados}

Otro objetivo de este artículo es analizar la distancia existente entre las motivaciones que los estudiantes exigirán en sus trabajos, y por tanto les gustaría encontrar y aquellas que realmente creen que encontrarán. Este gap motivacional puede ser utilizado por los gerentes y directivos para conocer mejor tanto a los estudiantes (futuros trabajadores) como al resto de sus trabajadores, y con ello poder utilizar técnicas para tener más motivados a sus trabajadores, y así lograr un mejor clima laboral. Con un mejor clima laboral puede conseguirse el equilibrio entre la satisfacción laboral y el compromiso organizacional.

Para ello utilizaremos las tres preguntas que después describimos y compararemos así los tres factores más importantes (tanto los que deben darse para aceptar el trabajo), como los que creen que realmente se dan en la empresa. Se describen por igual las diferencias existentes entre lo que se debe dar para aceptar el trabajo en comparación con [los factores] que realmente creen que pueden encontrar en la empresa. 


\section{Las diferencias entre los primeros y últimos cursos}

El último objetivo es estudiar las posibles diferencias entre las motivaciones de los estudiantes de los primeros cursos y los de los últimos cursos. Pretendemos comprobar si realmente la importancia otorgada al dinero se ve modificada con el transcurrir de los cursos y si cambia su influencia en los factores motivadores.

En resumen, pretendemos con nuestro estudio arrojar un poco de luz, o más bien intentar aportar alguna novedad aunque modesta a los estudios precedentes en el camino para lograr comprender la importancia del dinero y su papel dentro del sistema retributivo de los trabajadores, con el fin último de intentar, como imaginamos que será el objetivo común de todos los investigadores en el campo, lograr crear un sistema retributivo generalizable para los trabajadores con independencia de su edad, género y país donde trabajen.

\section{Hipótesis}

- H1: El dinero tiene una influencia significativa en la estructura motivacional.

o H1a: El dinero no es el primer factor de motivación laboral

o H1b: El sexo condiciona significativamente la elección de un buen sueldo como factor motivacional.

o H1c: El ciclo condiciona significativamente la elección del dinero como factor motivador.

- H2: Existe una diferencia significativa entre los deseos del estudiante y lo que espera obtener en la empresa.

o H2a: Existe un gap o distancia significativa entre los factores que para el alumno son importantes y los que se deberían dar para aceptar el trabajo. 
o H2b: Existe un gap o distancia significativa entre los factores que para el alumno son importantes y los que cree que realmente encontrará en la empresa.

o H2c: Existe un gap o distancia significativa entre los factores que se deberían dar para aceptar el trabajo y los que cree que realmente encontrará en la empresa.

\section{Metodología}

Escogemos una muestra de 310 alumnos de la licenciatura de Administración y Dirección de Empresas, de edades comprendidas entre los 17 y los 43 años, siendo la media de 21,11 años. El $51,7 \%$ de los alumnos está cursando el primer ciclo mientras que el 48,3\% se encuentra en el segundo ciclo. Respecto al género, el $45,6 \%$ son varones mientras que el $54,4 \%$ son mujeres. De estos 310 alumnos el $82,8 \%$ son españoles, el 7,6\% son franceses y el $9,6 \%$ son de otras nacionalidades. Otro de los factores considerados como sociodemográficos que hemos analizado es el hecho de que los alumnos tengan experiencia laboral bien porque estén trabajando en la actualidad o bien porque lo hayan hecho con anterioridad, puesto que consideramos que este hecho puede tener cierta influencia sobre los diferentes factores motivacionales y, en especial, sobre la retribución. El 57,1\% no trabaja mientras que el $42,9 \%$ tiene experiencia laboral. Ese $42,9 \%$ se distribuye de la siguiente forma: el 29,9\% trabajan esporádicamente, el $10,3 \%$ trabaja media jornada y el 2,7\% trabaja a jornada completa. Otro factor que nos pareció interesante de analizar era el tiempo que esperan que transcurra hasta que encuentren su trabajo. El $73,47 \%$ espera que no transcurran más de seis meses desde que termine la carrera hasta encontrar su primer trabajo, el 24,13\% espera que transcurran entre 6 y 18 meses, un 2,1\% espera que transcurran entre 18 y 24 meses, mientras que, finalmente, el $0,3 \%$ espera que transcurran más de 24 meses. El sector en el que les gustaría trabajar es en el sector servicios, circunstancia que 
podemos comprobar con los siguientes datos: el 71\% quisiera trabajar en el sector servicios, el 12,6\% le gustaría trabajar en el sector industrial, el 4,8\% en la construcción, el 1\% en la agricultura y pesca $y$, finalmente, el 10,6\% en otros sectores. Consideramos también que la elección del tipo de sector podía influir en los factores motivacionales de forma significativa. El 60,4\% prefiere trabajar en el sector privado, el 30,9\% prefiere trabajar en el sector público $y$, finalmente, al 8,7\% le resulta indiferente el tipo de empresa en el que trabaja. Finalmente, les preguntábamos si les gustaría trabajar en el extranjero o no; obtuvimos como respuesta que al 51,2\% le gustaría trabajar en el extranjero, el 45,5\% no quiere trabajar en el extranjero mientras que al 3,3\% le resulta indiferente.

La razón por la cual escogemos alumnos de primeros y últimos cursos es para analizar si existen diferencias significativas entre las motivaciones laborales de los alumnos de los primeros cursos y los alumnos de los últimos cursos.

Las medidas que utilizamos para analizar la muestra fueron las siguientes (dos preguntas):

- Para medir los factores de la motivación utilizamos una escala Likert de 5 factores, a través de la cual el entrevistado debía valorar cada uno de los 15 factores motivacionales.

- Para medir los factores de la motivación utilizamos una escala Likert de 5 factores, a través de la cual el entrevistado debía valorar la medida en que cree que se producirá en la empresa cada uno de los 15 factores motivacionales.

Para escoger estos factores nos basamos en los que de antemano habían sido utilizados por (Luna-Arocas y Tang, 2004 y Harpaz, 1990) tal y como encontramos en nuestra revisión bibliográfica. Estos factores que los estudiantes debían valorar son: 


\begin{tabular}{|l|l|l|l|l|l|}
\hline \multirow{2}{*}{ Factores } & \multicolumn{5}{c|}{ Valoración } \\
\hline & 1 & 2 & 3 & 4 & 5 \\
\hline Buen sueldo & & & & & \\
\hline Posibilidad de promocionar & & & & & \\
\hline Más responsabilidad & & & & & \\
\hline Buenas condiciones salud/higiene en el trabajo & & & & & \\
\hline Estabilidad laboral & & & & & \\
\hline Buen clima laboral & & & & & \\
\hline Trabajo interesante & & & & & \\
\hline Aprendizaje con mi inmediato superior & & & & & \\
\hline Trabajo con retos personales & & & & & \\
\hline Formación & & & & & \\
\hline Horario adecuado & & & & & \\
\hline Autonomía & & & & & \\
\hline $\begin{array}{l}\text { Coincidencia entre los requerimientos laborales } \\
\text { y las habilidades y experiencia necesarias }\end{array}$ & & & & & \\
\hline Variedad de tareas & & & & & \\
\hline
\end{tabular}

Leyenda: 1. Completamente en desacuerdo

2. Bastante en desacuerdo

3. Bastante de acuerdo

4. Neutral

5. Completamente de acuerdo

Posteriormente, el alumno debía citar en tres preguntas diferentes los tres factores preferidos en cada caso. En la primera pregunta se les pedía que escogieran por orden aquellos tres factores que ellos encontrarían necesarios para poder aceptar un trabajo. En la segunda, se les pregunta en torno a los tres factores que deberían darse para aceptar el trabajo. En la tercera, se les pedía que citaran por orden de importancia aquellos factores que, a su entender, encontrarían en sus futuros trabajos. Con esta primera pregunta pretendemos ver si hay coherencia entre su valoración de cada uno de los factores motivacionales y la primera pregunta. Respecto a la segunda pregunta el objetivo es analizar el gap (distancia) entre los factores que esperan encontrar y los que realmente creen que encontrarán, ya que puede ser un factor a tener en cuenta por los 
directivos y gerentes no solo a la hora de contratar a un recién licenciado sino también a la hora de saber cómo incentivarlo o motivarlo.

\section{Resultados}

En primer lugar, uno de los primeros objetivos que nos marcamos era ver si se cumplía en el caso de los estudiantes el hecho de que un buen sueldo o remuneración tenga un papel relevante en la motivación de los empleados; a pesar de ese papel relevante existen factores que sin embargo tienen una mayor influencia en la motivación de los empleados. El resultado que obtuvimos fue que lejos de lo que pensábamos los estudiantes valoran en primer lugar un buen sueldo tanto como primer factor motivador en un $67,9 \%$ de los casos, siendo también el primer factor (con un 43,6\% que debería darse) para que acepten el trabajo.

En segundo lugar, analizamos cómo podía influir el género en la elección del primer factor motivador y obtuvimos como resultado que el $76 \%$ de los hombres puntúa la retribución en el primer lugar; tan solo el $61 \%$ de las mujeres lo situaban en primer lugar. En el caso del primer factor que se debería dar para aceptar el trabajo el 50\% de los hombres escogió un buen sueldo mientras que en el caso de las mujeres la cifra descendía hasta el 39\%.

Para realizar el análisis de la edad hemos tomado la edad original y atendiendo a criterios lógicos la hemos convertido en una variable dicotómica basándonos también en la posible experiencia laboral de los alumnos. En el primer grupo tomamos los alumnos de hasta 20 años que se cuya experiencia laboral se supone que debe ser escasa o incluso nula tal y como se confirmaba en nuestros datos y que correspondía con el 45,2\% de los casos, mientras que el segundo grupo corresponde a los alumnos de entre 20 y 42 años que tal y como comentábamos tienen una mayor experiencia laboral y que corresponden con un 54,8\% de los alumnos entrevistados. Del análisis de estos dos grupos obtuvimos como resultado que un $81 \%$

494 Ciencia y Sociedad 2014; 39(3): 477-508 
de los alumnos del primer grupo de edad (aquellos con menor experiencia laboral) eligen como primer factor motivacional el sueldo mientras que en el segundo grupo este porcentaje desciende hasta el 58\% de los casos. En el caso del primer factor que debería darse para aceptar el trabajo, el primer grupo de edad puntuó un buen sueldo, el 50\% de los casos seguido de unas buenas condiciones de salud/higiene laboral; un 10\% de los casos procura la estabilidad laboral; la posibilidad de promocionar y de un trabajo interesante con un $9 \%, 8 \%$ y $6,7 \%$, respectivamente. Mientras que el segundo grupo valoró un buen sueldo en un 39\% de los casos, buenas condiciones de salud/higiene laboral en un $20 \%$ de los casos, la posibilidad de promocionar en un $10 \%$ de los casos, un trabajo interesante en un $8,8 \%$ y la estabilidad laboral en un $7,5 \%$ como el primer factor que se debería dar para aceptar el trabajo.

Partiendo de los resultados obtenidos se confirma la hipótesis de que el ciclo condiciona significativamente la estructura motivacional de los estudiantes ya que mientras los alumnos del primer ciclo puntúan un buen sueldo como primer factor motivador en un $80 \%$, en el segundo ciclo tan solo el $54 \%$ de los estudiantes lo valora en primer lugar pasando a valorar también otros factores como pueden ser la posibilidad de promocionar o la estabilidad laboral.

La hipótesis que pretendía demostrar el hecho de que el trabajar o no condicionaba la estructura motivacional se confirma significativamente. El 62,5\% de los alumnos que trabajan escogen un buen sueldo como primer factor motivacional seguido de la posibilidad de promocionar en un 9,4\% de los casos y por la estabilidad laboral en un $8,6 \%$ de los casos. En contraposición los alumnos que no trabajan valoran un buen sueldo como primer factor en un $71 \%$ de los casos, seguido de la posibilidad de promocionar en un $8,8 \%$ de los casos y por la estabilidad laboral en un $6,5 \%$ de los casos. En el caso del primer factor que debería darse para aceptar el trabajo, el $37 \%$ de los que trabajan prefieren un buen sueldo 
mientras que en el caso de los estudiantes que no trabajan ese porcentaje aumenta hasta el $47 \%$ de los casos. Del grupo que trabaja, el 66\% de los casos eligió un buen sueldo en primer lugar, mientras que los que trabajan media jornada lo hicieron en un $62 \%$ de los casos y los que trabajan a jornada completa en un $25 \%$, con el que el descenso se muestra significativo.

También analizamos la distancia existente entre los factores que el estudiante desea y los que cree que en realidad se darán en la empresa, para ello hemos creado una variable nueva denominada gap estratégico. Observamos que la mayor distancia se produce entre el horario adecuado deseado (con una media de 4,38) y el que creen que realmente se dará con un 2,88 y cuyo gap es de 1,50, entre el trabajo interesante $(\mathrm{x} 4,39)$ y el que realmente se dará (x 2,92) con un gap de 1,47 y entre el buen sueldo deseado $(4,55)$ y el esperado $(3,10)$ con un gap de 1,45 . Los factores donde el gap es menor son entre la responsabilidad deseada $(3,85)$ y la esperada con un $(3,49)$ cuyo gap es de 0,36 .

También puede analizarse el gap tal y como proponemos en las hipótesis a través de la comparación entre los factores motivacionales deseados, los que deberían darse para aceptar el trabajo y los que realmente se esperan en la empresa. De esta comparación obtuvimos los siguientes resultados: en la comparación entre los factores deseados y los que deberían darse para aceptar el trabajo destacan de modo fundamental los siguientes: dos de cada tres personas consideran que el factor más importante es el dinero, no llega a dos de cada cuatro personas (44\%) la cantidad de estudiantes que requieren un buen sueldo para aceptar el trabajo; en el caso del gap entre los factores deseados y los que los estudiantes creen que realmente se darán en la empresa destaca el hecho de que tan solo $5,8 \%$ espera obtener un buen sueldo. Los factores motivacionales más esperados por parte de los estudiantes son unas buenas condiciones de salud/higiene laboral en un 31,5\% de los casos, seguido de la formación, elegido como factor que los estudiantes consideran que se da con mayor frecuencia, en el 12,7\% de los casos. 
Tal y como explicamos en la introducción uno de nuestros principales objetivos era ver si se podían agrupar de alguna manera los factores motivacionales en función de las respuestas obtenidas de los estudiantes con la finalidad de poder establecer mecanismos generalizables de motivación, y con ello abastecer a los directivos de recursos humanos de una herramienta útil a la hora de contratar a los actuales estudiantes en un futuro.

Para ello realzamos un factorial del cual obtuvimos cuatro grupos de factores; un primer grupo compuesto por las condiciones de higiene/salud en el trabajo, la estabilidad laboral, un horario adecuado y un buen clima laboral, un segundo grupo formado por un trabajo interesante, autonomía, variedad de tareas y la coincidencia entre los requerimientos laborales y las habilidades y experiencia necesarias; un tercer grupo formado por una mayor responsabilidad, un aprendizaje con el inmediato superior y la formación, un cuarto y último grupo formado por un buen sueldo y la posibilidad de promocionarse. Al realizar la prueba de fiabilidad obtuvimos valores del 0,623, 0,675, 0,666 y 0,309 para cada uno de los grupos, respectivamente.

A pesar de que se pueda tal vez justificar mediante el trabajo de Nunnally las fiabilidades obtenidas para los tres primeros grupos, desde la lógica consideramos que estos tres grupos no pueden justificarse ya que no podemos establecer un paralelismo entre factores intrínsecos y extrínsecos y tampoco tienen excesiva lógica los grupos ya que desde nuestro punto de vista los factores son suficientemente explicativos por sí mismos. Por lo que, en realidad, no alcanzamos nuestro objetivo de intentar establecer o encontrar grupos de factores que explicasen en mejor medida la actitud de los trabajadores y su compromiso para con la organización.

Otro de nuestros objetivos era intentar establecer grupos con comportamientos comunes, de tal forma que se simplificase la tarea de los directivos a la hora de comprender el comportamiento de los futuros trabajadores y poder actuar más fácilmente con ellos. 
Con la finalidad de alcanzar este objetivo realizamos un clúster realizando primero un análisis a través de los conglomerados jerárquicos intentando analizar a través del dendograma, y sobre todo del coeficiente de fusión como método de comprobación, para determinar cuántos grupos se formaban y, posteriormente, aplicar la técnica de conglomerados por $\mathrm{K}$ medias. Aplicando estas técnicas describimos tres grupos más o menos heterogéneos. En un primer análisis se obtuvo un primer grupo que puntúa todos los factores por encima de los otros dos. Los otros dos grupos se caracterizan por lo siguiente: uno por valorar, en mayor medida, un horario adecuado, un buen sueldo, la estabilidad laboral y la coincidencia entre los requerimientos laborales y las habilidades y experiencia necesarias, mientras que el otro se caracteriza por otorgar una mayor importancia a la responsabilidad, al trabajo interesante, al trabajo con retos personales y a la variedad de tareas sin importarle tanto el dinero.

En un análisis hecho grupo por grupo, o sea, de carácter más pormenorizado destaca el primer grupo ya que le da una mayor importancia a las condiciones extrínsecas del puesto de trabajo, en este aspecto destaca su deseo por obtener un buen sueldo, un buen horario, buen clima y estabilidad, sin importarle tanto la variedad de tareas, la autonomía, el aprendizaje, entre otros factores intrínsecos. Parece que este tipo de estudiante busca un puesto de trabajo donde se consolide con un buen sueldo y se encuentre a gusto sin intentar promocionar[se], y dándole una menor importancia a la variedad de tareas dentro del puesto de trabajo aunque cabe no olvidar que el estudiante de este grupo valora todos los factores como bastante o muy importantes. El primer grupo está formado por 166 estudiantes que corresponden con el 56\% de los estudiantes dividido en un 35\% de hombres y un $65 \%$ de mujeres. El $43 \%$ de sus miembros tiene experiencia laboral, el $56 \%$ de sus miembros prefiere trabajar en una empresa privada, mientras que el $54 \%$ no le importaría trabajar en el extranjero. 
Este grupo es el escogido por el $57 \%$ de los españoles mientras que tan solo el 25\% de los franceses escogería este grupo.

El segundo grupo destaca fundamentalmente por darle mucha importancia al dinero sin importarle tanto el resto de factores mencionados. Está compuesto por 98 estudiantes que se corresponden con un $33 \%$ de los estudiantes encuestados, repartidos en un $60 \%$ de hombres y un $40 \%$ de mujeres, de los cuales el $91 \%$ son españoles y siendo el segundo grupo más escogido por los españoles. El 52\% de sus miembros tiene experiencia laboral. Al $62 \%$ de sus miembros le gustaría trabajar en una empresa privada; a diferencia de los otros dos grupos a la mayoría de sus miembros no le gustaría trabajar en el extranjero $(63,1 \%)$.

A diferencia del primer grupo, el tercer grupo se caracteriza por darle una mayor importancia a los factores intrínsecos, como un trabajo con retos personales, donde se les dé una alta responsabilidad y se preocupan menos por los factores extrínsecos y relativos al puesto de trabajo excepto por un trabajo interesante ya que es el factor que más puntúan. Cabe también destacar el hecho de que no les otorgan tanta importancia a un buen sueldo, como los otros dos grupos. Este grupo se preocupa por su crecimiento personal más que por las condiciones del puesto de trabajo. Este grupo es el minoritario y está formado por 33 estudiantes, de los cuales el 63\% son hombres y el 37\% son mujeres. Este grupo es el más escogido por el colectivo de estudiantes franceses, lo escogen un $60 \%$ de sus miembros. El $31 \%$ de sus miembros tiene experiencia laboral con lo que podemos decir que es el único grupo de los tres formado mayoritariamente por gente sin experiencia laboral. Al 76\% de sus miembros le gustaría trabajar en una empresa privada, destacando el hecho de que a la mayoría de sus miembros les gustaría trabajar en el extranjero. Cabe destacar también que a diferencia de los dos anteriores este grupo está formado en su mayoría por miembros del segundo ciclo (74\%). 
A nivel general destaca el hecho de que los tres grupos ponderan por igual la posibilidad de promocionarse, aunque en términos relativos un grupo le da más importancia que el otro. También es importante el hecho de que en ninguno de los tres grupos se le otorga mucha importancia en términos relativos al aprendizaje con el inmediato superior. Finalmente, respecto al ciclo es interesante ver cómo en los dos primeros grupos no tiene excesiva trascendencia puesto, puesto que se reparten más o menos por igual, mientras que en el tercer grupo la diferencia es significativa, tal y como comentábamos es mucho mayor la participación de miembros del segundo ciclo.

Cabe destacar que todas nuestras hipótesis se confirman exceptuando la hipótesis H1a ya que el dinero sí que es el primer factor motivacional.

\section{Conclusiones}

Para atraer, retener y motivar a los empleados los directivos necesitan aplicar una combinación de estrategias compensatorias (Milkovich \& Newman, 2002) y técnicas y factores motivacionales. Tanto los factores intrínsecos como los extrínsecos son muy importantes como Hertzberg demostró en sus artículos. En este artículo demostramos la importancia del dinero como moderador de la relación entre la satisfacción laboral y el compromiso organizacional.

También demostramos que hay una diferencia significativa entre los factores motivacionales que los estudiantes les gustaría encontrar y los que realmente están dispuestos a aceptar el estudio de estas diferencias puede ser una clave para los gerentes y directores de recursos humanos para poder contratar a los mejores estudiantes, o mejor dicho, a aquellos estudiantes con el mejor perfil, al igual que también será una herramienta motivacional potente.

El dinero es un factor motivador para algunas personas. Tanto los investigadores como los directivos necesitan tener un profundo 
conocimiento acerca de las motivaciones intrínsecas de los estudiantes como futuros trabajadores. Los directivos deberían tener cuidado con la estrategia que emplean con el dinero en las organizaciones. Algunos autores han encontrado limitaciones al dinero porque el mismo no es un factor motivador para todo el mundo ante en las mismas condiciones (Kohn, 1993), aunque en nuestro caso ha sido imposible demostrarlo, ahora bien analizando el tercer grupo, el dinero se convierte en el segundo factor motivacional por detrás del trabajo interesante.

Es necesario realizar mayores estudios y con mayor profundidad sobre la importancia del dinero (Mitchel y Mickel, 1999) en el contexto organizacional, para sustentar las estrategias aplicadas con los avances científicos. En síntesis, debemos utilizar el dinero de forma moderada en el sistema de compensación, sobre todo poniendo énfasis en que el dinero es un factor de recompensa extrínseca pero no debemos olvidar los factores intrínsecos. El dinero debe incluirse en la estrategia compensatoria pero no como el principal motivador laboral, ya que la motivación es mucho más compleja.

\section{Referencias bibliográficas}

Bass, B., \& Eldridge, L. (1979). Accelerated managers, objectives in twelve countries. Industrial Relations, 12, 158-71.

Bassett-Jones, N., \& Lloyd, G. C. (2005). Does Herzberg's motivation theory have staying power? The Journal of Management Development, 24(10), 929-930.

Beer, M., \& Katz, N. (2003). Do incentives work? The perceptions of a worldwide sample of senior executives. Human Resource Planning, 26(3), 30-35.

Centres, R., \& Bugental, P. (1966). Intrinsic and extrinsic job motivations among different segments of the working population. Journal of Applied Psychology, 50, 193-97. 
Cheng, C. (2007). A Research study of Frederick Herzberg's motivator-hygiene Theory. Joumal of American Academy of Business, 12(1), 186-190.

Crosby, F. (1982). Relative deprivation and working women. New York: Oxford University Press.

Dowling, P. J., \& Nagel, T. W. (1986). Nationality and work attitudes: A study of Australian and American business majors. Journal of Management, 12, 121-28.

Dunn, S. (2001). Motivation by project and functional managers in matrix organizations. Engineering Management Journal, 13(2), 3-8.

Friedlander, F. (1965). Relationships between the importance and satisfaction of various environmental factors. Journal of Applied Psychology, 49, 160-164.

Garrido, M. J., Pérez, P., \& Antón, C. (2005). Determinants of sales manager job satisfaction. An analysis of spanish industrial firms. International Journal of Human Resource Management, 16(10), 1934-1954.

Groot, W., \& Van den Brink (1999). Job satisfaction of older workers. International Journal of Manpower, 20(6), 343-360.

Haire, M., Ghiselli, E. E., Porter, L. W. (1966). Managerial thinking: An international study. New York: Wiley.

Hardin, E., Reif, H., \& Heneman Jr., H. (1957). Stability of job preferences of department stores employees. Journal of Applied Psychology, 35, 256-259.

Harpaz, I. (1990). The importance of work goals: an international perspective. Journal of International Business, 21(1), 75-93.

Heneman, R. L. (1992). Merit pay. Massachusetts: Addison-Wesley. 
Herzberg, F. (1968). One more time: How do you motivate employees? Harvard Business Review, 52(5), 70-80.

Hertzberg, F., Mausner, B., \& Snyderman, B. (1959). The motivation to work". New York: John Wiley \& Sons.

Hinrichs, J. R. (1968). A replicated study of job satisfaction dimensions. Personnel Psychology, 21, 479-503.

Hofstede, G. H. (1972). The color of collars. Columbia Journal of World Business, 7, 72-80.

Igalens, J., \& Roussel, P. (1999). A Study of Relationships between Compensation Package: work motivation and job satisfaction, Journal of organizational behavior, 20, 1003-1025.

Jurgensen, C. E. (1978). Job preferences (what makes a job good or bad?". Journal of Applied Psychology, 63, 267-276.

Kannungo, R. N. (1983). A cross cultural comparative study of managerial job attitude. Journal of International Business Studies, 14(2), 115-129.

Kilpatrick, F., Cummings, Jr., M., \& Jennings, M. (1964). Source book of a study of occupational values and the image of the federal service. Washington: Brookings Institute.

Kohn, A. (1993). Why incentive plans cannot work. Harvard Business Review, 71, 54-63.

Kraut, A. I., \& Ronen, S. (1975). Validity of job facets importance: A multinational, multicriteria study. Journal of Applied Psychology, 60, 671-677.

Lawler, E. E. (1971). Pay and organizational effectiveness; A psychological view". New York: McGraw-Hill.

Lawler, E. E. (1973). Motivation in work organizations. Monterrey: Brooks/Cole. 
Leach, F., \& Westbrook, J. (2000). Motivation and job satisfaction in one government research and development. Engineering Management Journal, 12 (4), 3-10.

Luna-Arocas, R., \& Tang T. L. P. (2004). The love of money, satisfaction and the Protestant Work Ethic: Money profiles among university professors in the USA and Spain. Journal of Business Ethics, 50(4), 329-354.

McShane, S. L., \& Von Glinow, M. A. (2013). Organizational behavior (6th Ed.)". Boston: McGraw-Hill/Irwin.

Milkovich, G. T., \& Newman, J. M. (2004). Compensation. New York: Irwin McGraw-Hill

Milkovich, G. T., Newman, J. M., \& Gerhart, B. (2011). Compensation (10th Ed.). Boston: Irwin/McGraw-Hill.

Mitchell, T. R., \& Mickel, A. E. (1999). The meaning of money: An Individual-Difference Perspective. The Academy of Management Review, 24(3), 568-578.

Pascual-Ivars, J. (2006). "Money as a motivation factor. The gap between expected motivations and real given motivations". Congreso de la International Association for Research in Economic Psychology. Paris.

Porter, L. W. (1964). Organizational patterns of managerial job attitudes. New York: American Foundation for Management Research.

Porter, L. W., \& Lawler, E. E. (1965). Properties of organizational structure in relation to job attitudes and job behaviour. Psychological Bulletin, 64, 23-51.

Quinn, R. P. (1971). What workers want: The relative importance of job facets to American workers. Michigan: Survey Research Center. 
Rathankoon, R., \& Ogunlana, S. (2003). Testing Herzberg's twofactor theory in the Thai construction industry. Engineering, Construction and Architectural Management, 10 (5), 333-341.

Ronan, W. W. (1970). Relative importance of job characteristics. Journal of Applied Psychology, 54, 192-200.

Rynes, S. L., \& Gerhart, B. (2000). Compensation in organizations: Current research and practice. San Francisco, CA: Jossey Bass.

Rynes, S. L., Gerhart, B. \& Minette, K. A. (2004). The importance of pay in employee motivation: discrepancies between what people say and what they do. Human Resource Management, 43(4), 381-394.

Sauser, W. I., \& York, C. M. (1978). Sex differences in job satisfaction: A re-examination. Personnel Psycbology, 31, 537-547.

Sardžoska, E., \& Tang, T. L. P. (2012). Work-related behavioral intentions in Macedonia: Coping strategies, work environment, love of money, job satisfaction, and demographic variables". Journal of Business Ethics, 108(3), 373-391.

Sirota, D. y Greenwood, M. J. (1971). Understand your overseas work force. Harward Business Review, 49(1), 53-60.

Stracevich, M. M. (1972). Job factor importance for job satisfaction and dissatisfaction across different occupation levels. Journal of Applied Psychology, 56, 467-471.

Tang, T. L. P. (1993). The meaning of money: Extension and exploration of the Money Ethic Scale in a sample of University students in Taiwan", Journal of Organizational Behavior, 14, 93-99.

Tang, T. L. P., \& Liu, H. (2012). Love of money and unethical behavior intention: Does an authentic supervisor's personal integrity and character (ASPIRE) make a difference?" Journal of Business Ethics, 107(3), 295-312. 
Tang, T. L. P., Luna-Arocas, R., Quintanilla Pardo, I., \& Tang, T. L. N. (2012). Materialism and the bright and dark sides of the financial dream in Spain: The positive role of money attitudes - The matthew effect. Applied Psychology, an International Review. doi: 10.1111/j.1464-0597.2012.00533.x

Tang, T. L. P. Luna-Arocas, R., \& Sutarso, T. (2004). From income to pay satisfaction. The Love of Money and Pay Equity Comparison as Mediators and Culture (the United States and Spain) and Gender as Moderators. Management Research, 3(1), 7-26.

Tang, T. L. P., Luna-Arocas, R., Sutarso, T., \& Tang, D. S. H. (2004). Does the love of money moderate and mediate the income-pay satisfaction relationship?" Journal of Managerial Psychology, 19(2), 111-135.

Tang, T. L. P., Sutarso, T., Akande, A., Allen, M. W., Alzubaidi, A. S., Ansari, M. A., Arias-Galicai, F. et al. (2006). The love of money and pay level satisfaction: Measurement and functional equivalence in 29 geographical entities around the world. Management and Organization Review, 2, 423-452.

Tietjen, M., \& Myers, R. (1998). Motivation and job satisfaction. Management Decision, 36(4), 226-231.

Vroom, V. H. (1964). Work and motivation. New York: John Wiley and Sons.

Weiss, D. J., Dawis, R. V., England, G. W., \& Lofquist, L. H. (1964). Minnesota Studies in Vocational Rehabilitation. (VIII Construct validation studies of the Minnesota importance questionnaire). Bulletin 41, (s. p.).

Williston, B. (2005). Reasons for action and the motivational gap. The Journal of value inquiry, 39, 309-324. 


\section{José Vicente Pascual Ivars}

Es licenciado en Ciencias Económicas y Empresariales, doctor en Dirección de Empresas, Estrategia y Organización por la Universidad de Valencia, España. Es Diplom Kaufmann y Diplom Betriebswirtshaft und Internationalles Mangement por la Universidad de Bremen, Alemania. Obtuvo el título de máster en Gestión del Talento en 2009.

Es profesor asociado del Departamento de Dirección de Empresas desde el año 2005. Su especialización docente e investigadora se centra en las áreas de recursos humanos y emprendimiento.

Email: jose.pascual@uv.es

\section{Roberto Luna Arocas}

Es catedrático de Organización de Empresas de la Universidad de Valencia, España; profesor del Departamento de Dirección de Empresas de la Universidad de Valencia; presidente AECOP-EMCC España, Asociación europea de Coaching Ejecutivo; director del máster en Gestión del Talento. Es además presidente de AEDIPE CV.

Ha publicado los textos: El líder no nace... se hace: Viaje hacia el talento (Ed. Obelisco, 2010); en su nuevo libro Despierta el talento (Lid Editorial, 2011) nos muestra ocho ángulos diferentes para desarrollar el talento, esto lo hace a través de monólogos, 
historias y experiencias. Es coautor del libro Recursos bumanos en el turismo (Pearson Prentice Hall, 2007). Su principal área docente e investigadora se centra en los recursos humanos y en la gestión del talento.

Email: Roberto.luna@uv.es

Recibido: 07/03/2014 Aprobado: 17/08/2014 